\title{
Perceiving depth in point-light actions
}

\author{
JAN VANRIE and KARL VERFAILLIE \\ Katholieke Universiteit Leuven, Leuven, Belgium
}

\begin{abstract}
The present study investigates how observers assign depth in point-light figures, by manipulating spatiotemporal characteristics of the stimuli. Previous research on the perception of point-light walkers revealed bistability (i.e., that a point-light walker is perceived as either facing the viewer or facing away from the viewer) and the presence of a perceptual bias (i.e., a tendency to perceive the figure as facing the viewer). Here, we study the generality of these phenomena by having observers indicate the global depth orientation of different ambiguous point-light actions. Results demonstrate bistability for all actions, but the presence of a preferred interpretation depends strongly on the performed action, showing that the process of depth assignment takes into account the movements the point-light figure performs. Two additional experiments, using unfamiliar movement patterns without strong semantic correlates, show that purely kinematic aspects of an action also strongly affect depth assignment. Together, the results reveal the perception of depth in point-light figures to be a flexible process involving both bottom-up and top-down components.
\end{abstract}

Ever since Johansson (1973) introduced the point-light figure in biological-motion research, it has provided one of the most fascinating demonstrations of the perceptual system's sensitivity to actions performed by other animate beings. Observing nothing more than a swarm of bright dots indicating the major joints of a person suffices to convey the impression of that person engaged in a specific action (see, e.g., Ahlström, Blake, \& Ahlström, 1997; Cutting \& Kozlowski, 1977; Dittrich, 1993). Although much progress has been made in the study of computational and neural mechanisms underlying the perception of biological motion in general, and of point-light figures in particular (see Giese \& Poggio, 2003, for an overview), one issue that has been largely ignored is the perception of depth in point-light stimuli and of the overall orientation of the figure (for two exceptions, see Proffitt, Bertenthal, \& Roberts, 1984, and Verfaillie, De Troy, \& Van Rensbergen, 1994). Consider a 2-D point-light walker, as in Figure 1: Although the animated version of such a 2-D stimulus could have been the projected image of an infinite number of possible 3-D configurations, it is typically interpreted as the projection of a 3-D human walker. However, due to the unspecified depth order, resulting from the lack of ordinal depth cues, two such "human walker" percepts are in fact possible: one that is oriented toward the viewer and one away from the viewer. An everyday analogue of this

\footnotetext{
This research was supported by the Research Training Network "Perception for Recognition and Action" (Contract No. HPRN-CT-200200226) under the direction of the European Community and by the Fund for Scientific Research of Flanders, of which J.V. is a research assistant. The authors thank Martin Giese and two anonymous reviewers for very helpful comments on an earlier version of this article. Correspondence concerning this article should be sent to K. Verfaillie, Laboratory of Experimental Psychology, Katholieke Universiteit Leuven, Tiensestraat 102, B-3000 Leuven, Belgium (e-mail: karl.verfaillie@psy.kuleuven.be).
}

phenomenon can sometimes be experienced when one is observing people from a distance that is sufficient so that the people's internal features (such as facial elements or texture of clothing) are rendered uninformative. Although such an impoverished stimulus is typically highly recognizable ("Somebody's walking there ... .), the orientation in depth often remains ambiguous ("... but is he or she approaching or going away?").

Vanrie, Dekeyser, and Verfaillie (2004) investigated this issue and showed that point-light walkers seen from different viewpoints are perceived as 3-D human bodies in particular depth orientations and that a single point-light walker can indeed yield two different percepts, making it perceptually ambiguous or bistable (cf. Attneave, 1971). In addition, it was shown that despite the fact that both percepts are equiplausible, they are clearly not equiprobable: Observers show a strong tendency to interpret a pointlight walker as facing them. The difference in response frequencies for the two veridical interpretations was taken as evidence for the existence of a perceptual bias, and suggested that the mechanism underlying the interpretation of ambiguous point-light walkers is not simply a random process.

The present study asks how observers perceive the depth orientation of point-light figures in general. More specifically, we investigate whether the process of depth assignment depends on the spatiotemporal characteristics of the figure - that is, on the action that is performed. On the one hand, the specific movements of the pointlight figure might not be utilized at all in determining the depth order. For example, resolving the depth ambiguity of point-light figures might just be a stochastic process, in which the a priori probability of a facing interpretation is simply much higher (see, e.g., Domini \& Caudek, 2003; Hogervorst \& Eagle, 1998; Mamassian \& Landy, 1998). That is, for some reason - for example, the higher 


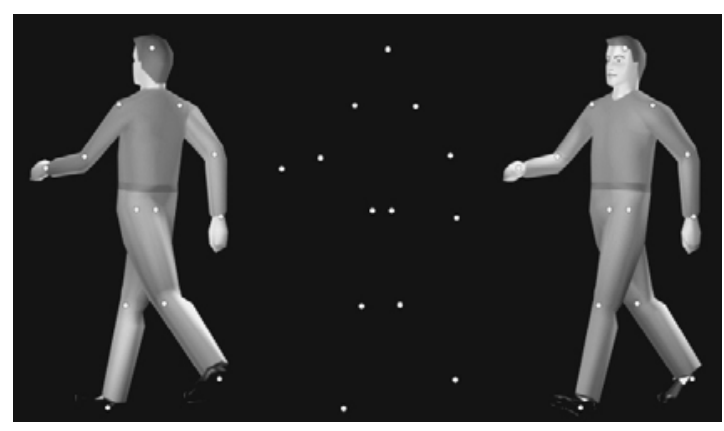

Figure 1. Single frame of a $3 / 4$ view of a point-light walker and the two veridical interpretations, with the point lights superimposed.

social importance of a facing orientation - there exists a general preference to perceive human bodies as being oriented toward the viewer. ${ }^{1}$ As a result of this, any ambiguity would generally be resolved by interpreting all stimulus features to be consistent with a facing interpretation, regardless of the specific movement characteristics of the body. In other words, the action the point-light figure performs does not contribute to the resolution of the ambiguity concerning the depth orientation and will thus not significantly affect the interpretation. On the other hand, contrary to this "general tendency" hypothesis, both the bistability and the perceptual bias might be consequences of a more stimulus-driven process, making these phenomena specific for the kinematics of a walking movement. It is known that the perceived identity of an ambiguous figure can influence the perceived movement direction of that figure (McBeath, Morikawa, \& Kaiser, 1992), and the influence of motion direction on the perceptual identification of ambiguous figures has also been shown (Bernstein \& Cooper, 1997). Likewise, the nature of the action performed might have an influence on the orientation in which the actor is perceived. In this "action-dependent" hypothesis, then, the computation of depth order of an ambiguous point-light figure would critically depend on the stimulus characteristics at hand.

\section{EXPERIMENT 1}

To investigate the process of depth assignment, we asked observers to indicate the global orientation of various ambiguous point-light actions. The predictions derived from the two hypotheses described above are both straightforward and opposite. The general tendency hypothesis states that whenever a human body is present, it is preferentially interpreted as facing the viewer. This leads to the prediction that, regardless of the action the point-light figure performs, the probability of a "facing the viewer" interpretation will be more or less the same. On the other hand, the action-dependent hypothesis would predict that, given a sufficiently broad sample of human actions, different results will be obtained for different actions, because the information present in these different patterns will be used by the visual system to determine the depth order.

\section{Method}

Participants. Twelve undergraduate students participated for course credit. All had normal or corrected-to-normal vision and were naive with respect to the purpose of the study, and none of them had previously been involved in studies with ambiguous pointlight figures.

Point-light configuration. The 3-D point-light configuration consisted of 13 markers indicating the center of the major joints of a male person and was projected orthographically onto the screen to create the actual 2-D stimulus (see Dekeyser, Verfaillie, \& Vanrie, 2002, for more details). All explicit depth cues were removed from the 2-D stimulus, there was no occlusion, and point lights had a constant size and luminance. Individual points subtended approximately $0.14^{\circ}$ of visual angle and were white against a black background. The size of the point-light figure itself was approximately $1.8^{\circ} \times$ $7.8^{\circ}$ of visual angle.

Design. Two independent variables were manipulated: the action performed (with four levels: jumping, rowing, cycling, and saluting) and the presented viewpoint (with five levels: two lateral views, one to the left and one to the right, two $3 / 4$ views, one to the left and one to the right, and a frontal/back view). The two variables were crossed, so that each action was shown from each of the five viewpoints. The participants performed all trials with the same action in one block, so that each participant experienced four blocks. Within each block, there were 10 repetitions of each of the five views. The order of trials within a block was randomized and the order of blocks was counterbalanced over participants.

Actions. Four movement patterns were selected from an action database (Vanrie \& Verfaillie, 2004; also see ppw.kuleuven.be/labexppsy/ actiondb.htm): an aerobic-style jumping movement, a cycling action consisting of two full revolutions, a rowing motion, and a onehanded saluting movement (see Figure 2), with durations of 1,200, $2,333,2,166$, and 3,166 msec, respectively. Two cycles of the jumping and rowing actions were shown, ${ }^{2}$ so the actual durations for these two stimuli were 2,400 and $4,332 \mathrm{msec}$. The actions were not intended to be a representative sample of all possible human actions, but rather a small collection of movements that nonetheless differ on several accounts: being more or less symmetrical (or not), implying global motion (or not), involving an object (or not), and so on.

Procedure. All of the participants were tested individually. They were seated in front of a 17-in. CRT in a dimly lit room. Viewing distance was $80 \mathrm{~cm}$. The participants were told that they would be presented with figures consisting of point lights (a lateral view of a point-light walker was shown as a demonstration) and that they were to indicate how these figures were oriented in depth - that is, which direction they were facing. A participant was to answer using a movable arrow attached to the center of a circle that was drawn on a cardboard sheet lying horizontally in front of him or her. The eight response alternatives were marked by eight lines originating from the center of the circle. A participant could indicate the perceived depth orientation by rotating the arrow about the center point so that it pointed toward one of the eight directions (e.g., the 6 o'clock position for a frontal/back view facing the viewer, the 12 o'clock position for a frontal/back view facing away, the 3 o'clock position for a lateral view to the right, and so on; cf. Vanrie et al., 2004). Of the eight possible responses, there were two correct responses for each nonlateral view: one orientation facing the viewer and one facing away from the viewer. It was stressed that an equal distribution of all eight orientation directions was not necessary. Upon seeing the stimulus, the participants could respond immediately, and the experimenter recorded the responses. The stimulus was followed by a black screen for $2 \mathrm{sec}$, after which a new trial started. Between the different 50-trial blocks, the participants had a short break.

\section{Results and Discussion}

Observers indicated the depth orientation of various point-light actions as seen from different viewpoints. The 


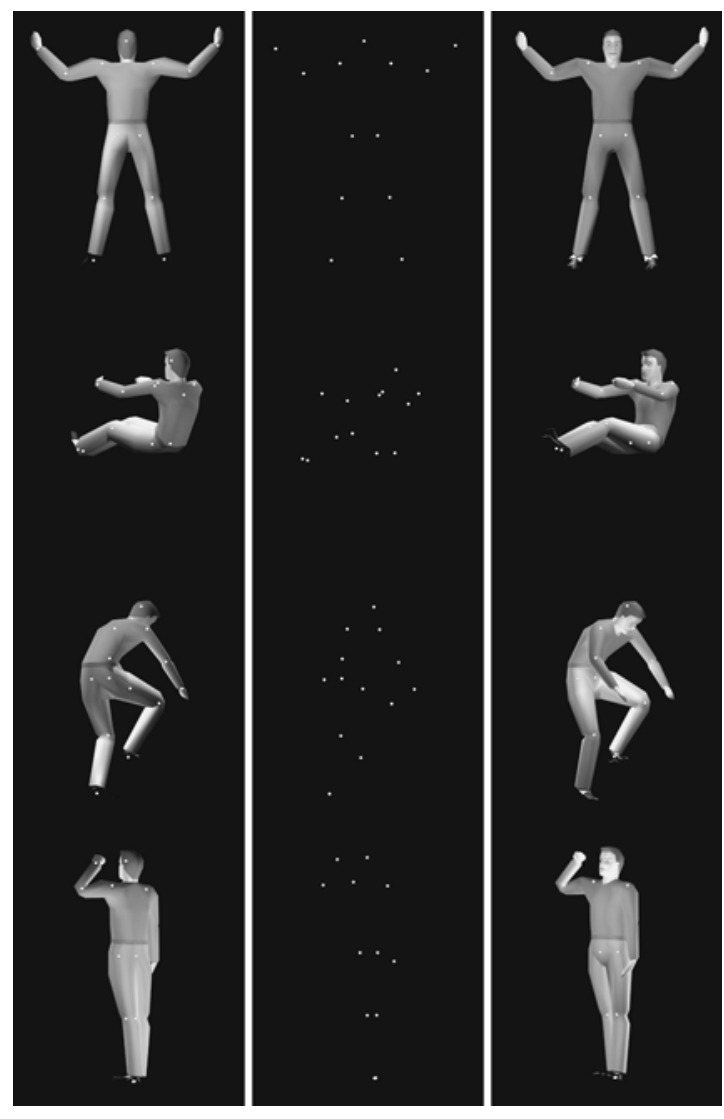

Figure 2. Single frame of each of the point-light actions in $\mathbf{E x}$ periment 1 and the two veridical interpretations, with the point lights superimposed. From top to bottom: jumping (frontal/back view), rowing ( $3 / 4$ view left), cycling ( $3 / 4$ view right), and saluting ( $3 / 4$ view left).

overall proportion of correct responses was .76 (chance level is .20). As illustrated in Figure 3, which shows the cumulative frequency of the different responses over all observers for the lateral, $3 / 4$, and frontal/back views of the four actions, observers generally reported a veridical orientation for the figure. However, for the $3 / 4$ views, there were also a large number of frontal/back interpretations reported. These errors can, in large part, be attributed to nonveridical reports for the saluting action. In this action, the visual differences between the $3 / 4$ and frontal/back views were actually so small that observers consistently interpreted all nonlateral views as frontal views: The two frontal responses (i.e., the one facing the viewer and the one facing away) accounted for $96 \%$ of the responses on all trials with a $3 / 4$ view. For this reason, and, more important, because we were primarily interested in whether the stimulus was perceived as "facing the viewer," as opposed to "facing away from the viewer," the following analyses were performed on all trials in which a nonlateral view was presented - that is, both correct and incorrect trials, ${ }^{3}$ except those trials in which a lateral response was given $(7.2 \%)$. The latter type of trial was not included in the analysis because it obviously does not fit the "facing the viewer" versus "facing away" dichotomy.

For all actions and per participant, we calculated the proportion of responses implying an orientation toward the observer ("facing the viewer" responses) across the nonlateral views (frontal/back and $3 / 4$ view). Now, when the stimuli are not perceptually ambiguous, we would expect a proportion of 1.00 (the figure is always perceived as facing the viewer) or 0 (the figure is never perceived as facing the viewer). On the other hand, when the two interpretations are equiprobable - that is, the figure is perfectly bistable-we would expect a proportion of .50. A facing bias (i.e., a tendency to interpret the figure as facing the viewer) would then be indicated by a proportion that was different from 1.00 but significantly above .50 .

A two-way repeated measures ANOVA with action and viewpoint (frontal/back vs. $3 / 4$ view) as independent variables showed a significant effect of the portrayed action $[F(3,33)=8.440, p<.001]$ but not the presented viewpoint $(F<1)$. The interaction $[F(3,33)=2.827, p=$ .054] was marginally significant. Means can be seen in Figure 4. Over viewpoints, we find a significant facing bias for the rowing and cycling actions (i.e., the point of equiprobability falls outside the $95 \%$ confidence interval), but not for the jumping and saluting actions.

The results provide a clear answer for the questions concerning the generality of bistability and perceptual bias. First, the perceptual ambiguity was found for all actions used in the experiment. Viewing a single point-light stimulus without explicit depth cues, regardless of the actual movements, results in naive observers reporting at least two different interpretations. This demonstrates that the visual processing of point-light actions in general, and not only of point-light walkers (cf. Vanrie et al., 2004), is sensitive to the ambiguities in the input, and it confirms the status of the point-light figure as being ambiguous or a bistable stimulus (Attneave, 1971). Second, a tendency to interpret a point-light figure as being oriented toward the viewer was not found for all movement patterns, and even for the patterns for which a higher proportion of "facing the viewer" responses was found, the strength of this facing bias varied. We therefore conclude that although certainly not unique for a walking movement, the facing bias is not an essential aspect of the perception of ambiguous point-light figures. It is important to note that this does not rule out the possibility of a general preference to view human bodies in a particular manner. A high-level heuristic - similar to, for example, the assumption that a light source is located above an object (see, e.g., Enns \& Rensink, 1990) - to interpret human bodies as being oriented toward the viewer might still play a role in the interpretation of the point-light walker and of point-light figures in general. However, the present results do indicate that such a general preference is not the sole determining factor in this process. Instead, the data indicate that in the resolution of the depth ambiguity, the information provided through the spatiotemporal characteristics of the stimulus is in fact being used. 


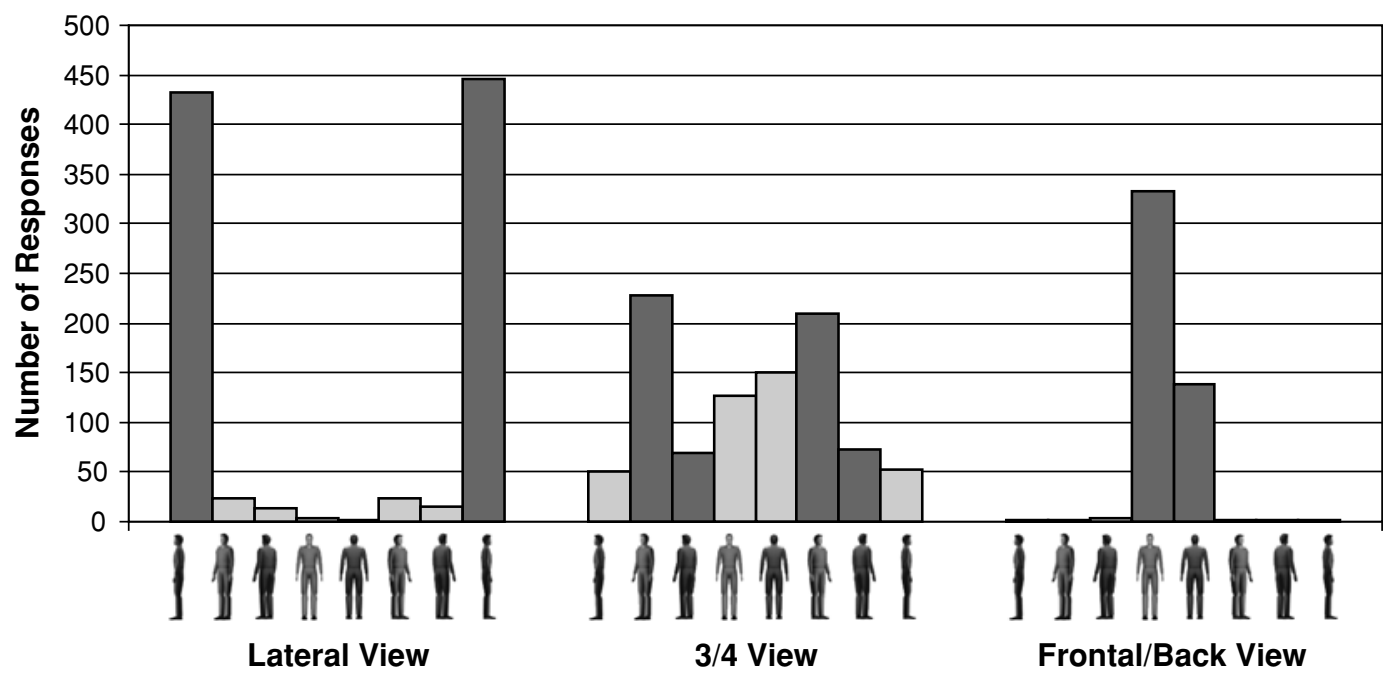

Figure 3. Histogram showing the frequency of reported orientations over observers and over the four actions as a function of the presented view (a lateral view, $a^{3} / 4$ view, and a frontal $/ \mathbf{b a c k}$ view). The different bars represent the eight possible responses (indicated by the different full-body images: from left to right, respectively, lateral to the left, $a^{3 / 4}$ view to the left facing the viewer, $a^{3} / 4$ view to the left facing away, a frontal view, a back view, $a^{3 / 4}$ view to the right facing the viewer, $a 3 / 4$ view to the right facing away, and a lateral view to the right). For each presented view, the veridical responses are indicated by the dark gray bars.

\section{EXPERIMENT 2}

The variation in both the presence and strength of the facing bias in the previous experiment supports an account in which the specific movements have an important bearing on the process of depth assignment in point-light stimuli. However, the actions used in that experiment differ on a number of features, both semantically and kinematically, ${ }^{4}$ all of which could, in principle, influence the determination of the global orientation. Moreover, these influences could also occur on different levels of processing in the visual system, depending on how the global orientation is actually computed. For example, if the visual system were to first process the action up to the point of identification, and only then resolve the orientation ambiguity, any effects of the kind of action would most likely be the result of high-level, top-down processes (cf. Bülthoff, Bülthoff, \& Sinha, 1998; Cavanagh, Labianca, \& Thornton, 2001). Alternatively, if the visual system starts by tracking the $3-\mathrm{D}$ motion of a single limb (i.e., two rigidly moving dots; cf. Webb \& Aggarwal, 1982), on the basis of which it reconstructs the complete body (as, for example, with the Necker cube; Kawabata, 1986), effects of the type of action will most likely reflect influences of lower-level, bottom-up processes.

The present experiment should be viewed as a first step in exploring the factors that mediate the process of

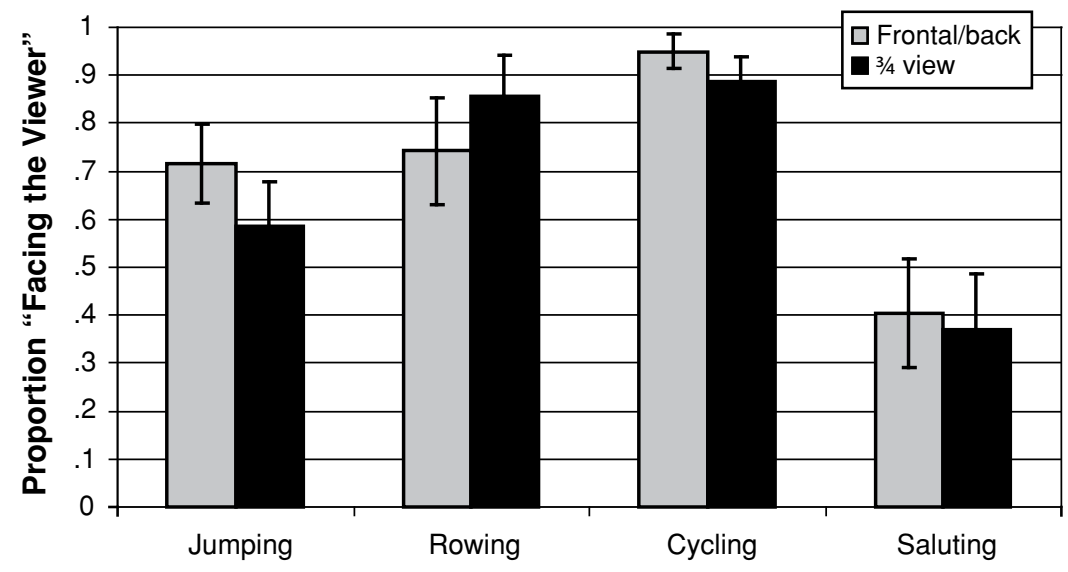

Figure 4. The proportion of responses in which observers in Experiment 1 reported the point-light figure in an orientation toward them for the frontal/back and $3 / 4$ view of the four actions (error bars denote the standard error). 
depth assignment. Here, we try to exclude semantically related effects as much as possible by including unfamiliar actions. That is, by using movements we assume are only rarely encountered, and that do not have an explicit goal or obvious verbal label, we attempt to minimize as much as possible effects of stored knowledge of actions (cf. "sprites"; Cavanagh et al., 2001). We certainly do not claim that these influences are totally irrelevant, but initially focus on the possible effects of specific aspects of the kinematic pattern of the point-light figure. The question is, of course, which kinematic features would be relevant for the process of depth assignment. As a starting point, we took a closer look at the movement pattern of those actions for which a high proportion of "facing the viewer" interpretations was previously found, and it revealed at least two common features - namely, the plane in which the upper limbs are located, vis-à-vis the torso, and the plane in which the lower limbs are located, vis-àvis the torso (see Table 1). More specifically, for walking, rowing, and cycling, both the arms and legs move or are located in front of the torso - that is, in a plane parallel to the midsagittal plane (e.g., while walking, the arms swing forward and backward), whereas for saluting and jumping, the limbs remain mostly in the same plane as the torso - that is, in the midfrontal plane (e.g., the sideways movements of arms and legs in the jumping pattern). Although we do not expect that these features are the only relevant ones or that they are the only determining factors of the results obtained in Experiment 1, we decided to replicate the previous experiment with four unfamiliar actions in which we could systematically manipulate the plane of movement of upper and lower limbs.

\section{Method}

Participants. Twelve naive undergraduate students with normal or corrected-to-normal vision participated for course credit. None of them had previously been involved in studies with ambiguous point-light figures.

Point-light configuration, Design, and Procedure. The basic setup was the same as in Experiment 1. We manipulated three independent variables: the direction of motion of the upper limbs (with two levels: lateral or perpendicular to the torso; see the Actions section for more details), the direction of motion of the lower limbs (with two levels: lateral or perpendicular to the torso), and the presented viewpoint (with the same five levels as in Experiment 1). Again, each of the four actions was shown from each of the five viewpoints.

Actions. Four action patterns were constructed, using the method described in Dekeyser et al. (2002). Briefly, a motion-capture system recorded the 3-D trajectories of 30 reflective markers that were attached all over the body of a human actor performing the different movements. The data from these markers were then used to animate a human-like skeleton in a graphical software package. Finally, by placing 13 virtual point-lights inside the major joints of this skeleton, we created the actual point-light stimuli (for a more detailed description, see Dekeyser et al., 2002).

In the four newly created action patterns, the plane of motion of both upper and lower limbs was systematically manipulated to be either lateral, vis-à-vis the torso, or perpendicular to it. The four patterns each consisted of a sequence of two movements in which the figure first took a step to one side and then, with the other leg, a step to the other side (i.e., lower limbs remained in the midfrontal plane, lateral to the torso) or the figure took a step forward and then, with the other leg, a step backward (i.e., lower limbs in a plane perpendicular to the torso, parallel to the midsagittal plane). During each of the two steps, the two outstretched arms made a simple swinging movement in phase (i.e., went up and down again simultaneously), also in a plane that was either parallel with the torso (i.e., to the sides of the body) or perpendicular to it (i.e., in front of the body [see Figure 5]; for dynamic demonstrations, see ppw.kuleuven.be/ labexppsy/biomotion.htm). Each pattern thus consisted of two steps and two arm movements, with a total duration ranging between 3,663 and $3,894 \mathrm{msec}$.

\section{Results and Discussion}

The overall proportion of correct responses was .86 (chance level is .20). For all four actions and per participant, we calculated the proportion of "facing the viewer" responses across the nonlateral views (frontal/back and $3 / 4$ view). As in the previous experiment, all trials in which a nonlateral view was presented were included, except those in which a lateral response was given $(1.1 \%) .{ }^{5}$

A three-way ANOVA with viewpoint (frontal/back vs. $3 / 4$ view), direction of upper limb movement (lateral vs. perpendicular to the torso), and direction of lower limb movement (lateral vs. perpendicular to the torso) as independent variables revealed three significant effects: the direction of upper limb movement $[F(1,11)=24.453, p<$ $.001]$, the interaction between viewpoint and direction of upper limb movement $[F(1,11)=31.152, p<.001]$, and the three-way interaction $[F(1,11)=7.552, p=.019]$.

On the basis of a rough analysis of the actions used in Experiment 1, we decided to manipulate two kinematic features in the present experiment: the direction of motion of the arms and the direction of motion of the legs. As is evident in Figure 6, the results can be summarized in three general findings. First, the plane of movement of the lower limbs is not the most relevant factor, because this, in itself, did not have a significant effect on the interpretation. Second, the kinematic pattern clearly does

Table 1

Summary of the Actions Used in Experiment 1 and of the Walking Action Used in Vanrie et al. (2004) in Terms of Presence of a Facing Bias and Locations of Both Upper and Lower Limbs Relative to the Torso

\begin{tabular}{llll}
\hline $\begin{array}{c}\text { Movement } \\
\text { Pattern }\end{array}$ & $\begin{array}{c}\text { Facing } \\
\text { Bias }\end{array}$ & $\begin{array}{c}\text { Upper Limbs } \\
\text { Relative to the Torso }\end{array}$ & $\begin{array}{c}\text { Lower Limbs } \\
\text { Relative to the Torso }\end{array}$ \\
\hline Rowing (Exp. 1) & yes & perpendicular & perpendicular \\
Cycling (Exp. 1) & yes & perpendicular & perpendicular \\
Saluting (Exp. 1) & no & lateral & lateral \\
Jumping (Exp. 1) & no & lateral & lateral \\
Walking & yes & perpendicular & perpendicular \\
\hline
\end{tabular}



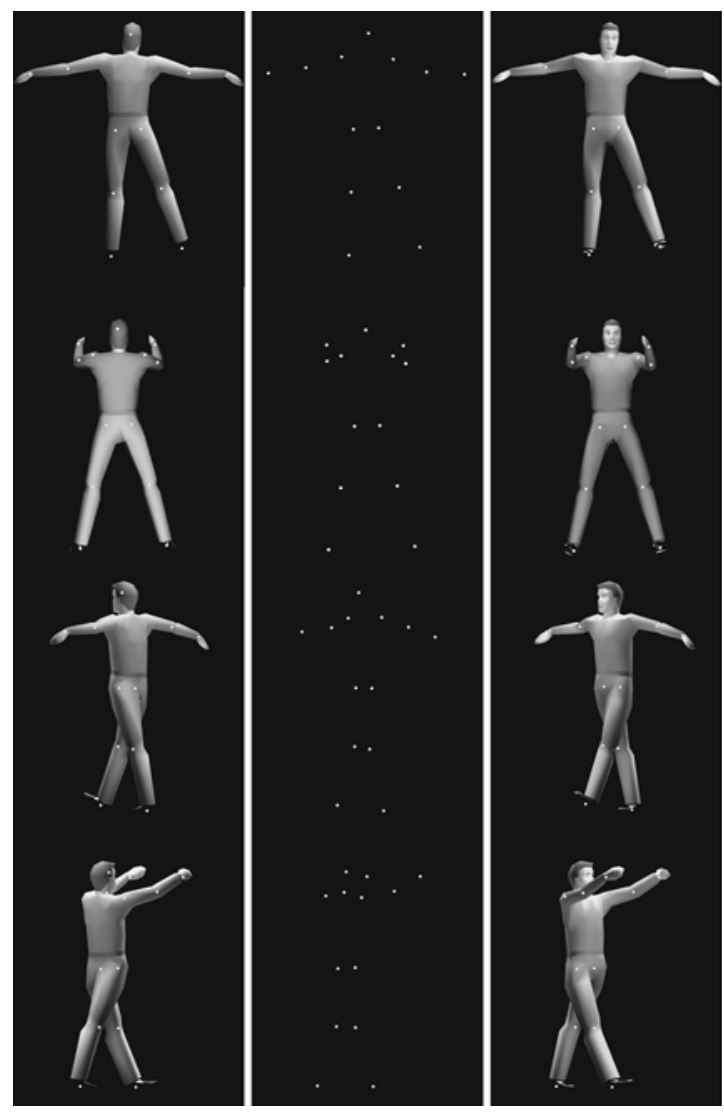

Figure 5. Single frame of each of the point-light actions in Experiment 2 and the two veridical interpretations, with the point lights superimposed. From top to bottom: upper limbs lateral and lower limbs lateral (frontal/back view), upper limbs perpendicular and lower limbs lateral (frontal/back view), upper limbs lateral and lower limbs perpendicular ( $3 / 4$ view left), and upper limbs perpendicular and lower limbs perpendicular ( $3 / 4$ view right).

have a strong effect on the depth assignment process. A preferred interpretation becomes apparent only when the upper limbs move perpendicular, vis-à-vis the torso. Third, as indicated by the significant interactions, the previous points do not hold in every instance, but seem to depend in part on the viewpoint from which the stimulus is presented. When the figure is presented from a frontal viewpoint, the preference to interpret the stimulus as facing the viewer, observed for $\mathrm{a} 3 / 4 \mathrm{view}$, is either diminished or completely absent.

Before discussing the results of this experiment in more detail, we first wanted to check the robustness and generality of the effects we observed, and we also wanted to try to clarify the influence of the specific viewpoint from which the stimulus is presented. Therefore, we decided to replicate the experiment with some specific changes.

\section{EXPERIMENT 3}

The previous experiment revealed some strong but not completely consistent effects of the specific movement characteristics on the perception of the global depth orientation of a point-light figure. To investigate the reliability of these effects, we repeated the experiment with two major changes.

First, given that the stimuli in Experiment 2 were blocked per action, observers were presented with several repetitions of the same stimulus in a single block. One could argue that this procedure introduces additional factors that somehow skew the results (e.g., it could encourage observers to actively search for other ways to interpret the stimulus; of course, this immediately raises the question of why this would occur much more frequently for some stimuli compared with others). In the present experiment, the different action patterns are presented in a mixed fashion, so that each stimulus - that is, each view from each movement pattern - is presented only once per block. The second change concerns the stimuli proper. In Experiment 2, the movement patterns consisted of two steps (forward and backward or to the sides) and two arm movements (perpendicular or lateral, vis-à-vis the torso). On the one hand, if the differential results are indeed primarily related to the direction of movement of the upper limbs, we should observe the same results for each individual step/arm movement. On the other hand, the relatively long duration and the fact that the stimuli in Experiment 2 are actually compounds (i.e., they consist of two sequential steps/arm movements) might have distorted the results. ${ }^{6}$ In the present experiment, only the single steps/arm movements are used.

\section{Method}

Participants. Twelve naive undergraduate students with normal or corrected-to-normal vision participated for course credit. None of them had previously been involved in studies with ambiguous point-light figures.

Point-light configuration. The same configuration was used as in Experiment 1.

Actions. The four action patterns in the previous experiment each consisted of a sequence of two arm/leg movements. Here, each animation of Experiment 2 was simply divided in two time segments of approximately equal length, so that both the first segment, showing a single arm/leg movement, and the second segment, showing the second step and arm movement, now constituted independent stimuli (albeit with the same features in terms of plane of movement of the upper and lower limbs). That is, the four original point-light animations-as used in Experiment 2 and showing two sequential arm/leg movements - were split up, yielding eight stimuli, each of which consisted of only one step and one arm movement. No other modifications were applied. Total duration of the stimuli was between 1,749 and $1,947 \mathrm{msec}$.

Design and Procedure. The basic design and procedure were the same as in the previous experiment, with two exceptions. Again, we had as independent variable the viewpoint (with five levels), the direction of movement of the upper limbs (lateral vs. perpendicular), and the direction of movement of the lower limbs (lateral vs. perpendicular). However, because the movement patterns of Experiment 2 were divided in two parts, we now have two instances of each of the four possible action patterns. A second change in comparison with Experiment 2 was that the different movement patterns were no longer presented in a blocked fashion. The eight stimuli were presented in a random order, in five blocks of 40 trials, so that in each block, each of the eight actions was presented once from each of the five viewpoints. 


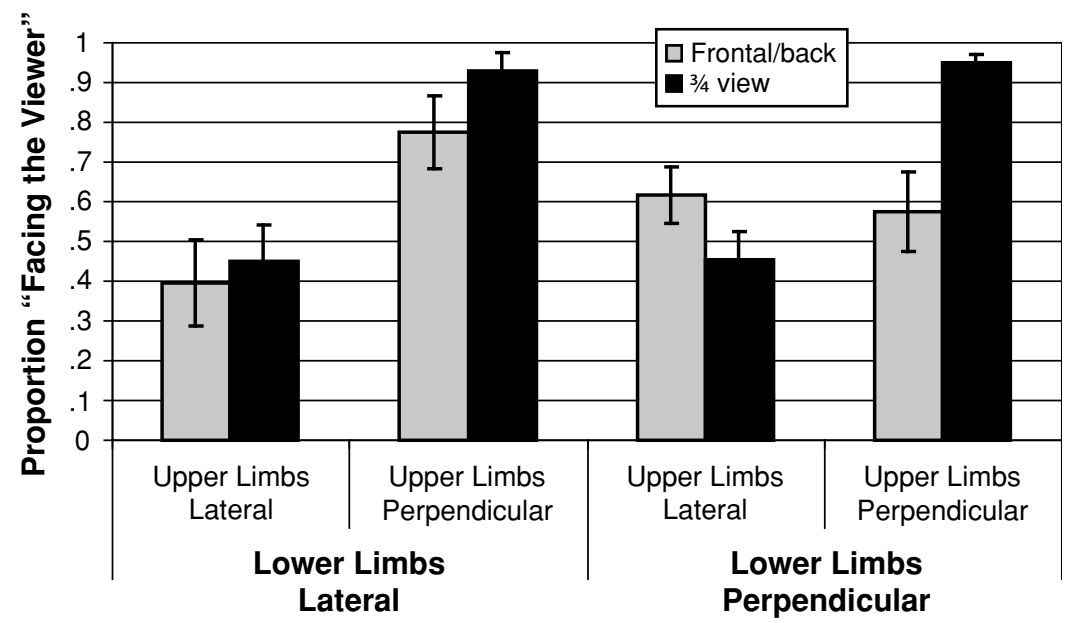

\begin{abstract}
Figure 6. The proportions of responses in which observers in Experiment 2 reported the point-light figure in an orientation toward them for the frontal/back and $3 / 4$ views of the four actions, classified in terms of the direction of movement (perpendicular or lateral) of the upper and lower limbs (error bars denote the standard error).
\end{abstract}

\section{Results and Discussion}

The overall proportion of correct responses was .69 (chance level is .20). Once again, for the eight actions and per participant, the proportion of "facing the viewer" responses across the nonlateral views (frontal/back and $3 / 4$ views) was calculated over all trials in which a nonlateral view was presented, except when a lateral response was given $(2.5 \%){ }^{7}$

The upper panel of Figure 7 shows the results for the four stimuli that correspond to the first time segment of the actions used in the previous experiment, and the lower panel depicts the results for the four stimuli corresponding to the second time segment. As can be seen by comparing the upper and lower panels, stimuli that correspond in terms of plane of movement of upper and lower limbs (i.e., that were a part of the same stimulus in the previous experiment) are also perceived in the same way. Consequently, the data for the corresponding stimuli were collapsed and analyzed in a three-way repeated measures ANOVA, with viewpoint and plane of movement of both upper and lower limbs as independent variables. The analysis yielded significant main effects of the presented viewpoint $[F(1,11)=$ $8.919, p=.012]$ and the movements of the upper limbs $[F(1,11)=6.927, p=.023]$ and a significant interaction between these two factors $[F(1,11)=16.741, p=.002]$. No other effects approached significance $\left(F_{\mathrm{s}}<1\right)$.

Despite the changes in experimental paradigm and stimuli, in comparison with Experiment 2, the pattern of results observed here is very similar. There is, however, one distinction: The proportion of "facing the viewer" responses for the frontal viewpoint of the two actions with the upper limbs perpendicular and the lower limbs lateral has decreased, so that instead of a significant three-way interaction, a main effect of viewpoint was observed. One possible reason for this difference might be that because of the blocked presentation in Experiment 2, the fairly strong facing bias for the $3 / 4$ view generalized to the frontal/back view, although in itself, the frontal/back view would not elicit that many facing interpretations. The somewhat lower proportion of "facing the viewer" responses for frontal/back views in Experiment 2 is consistent with this hypothesis. In Experiment 3, then, generalization from a $3 / 4$ view to a frontal/back view of the same action would no longer occur due to the mixed presentation. As a result, the facing bias would also no longer be found for these views. Nevertheless, although the reason for this specific difference is not entirely clear, the general agreement between both experiments implies that the effects are indeed related to the stimulus manipulations that were used.

Concerning the role of the lower limb movements, the results confirm and extend those of Experiment 2: Pointlight figures stepping sideways, forward, or backward yield similar interpretations. The absence of an effect of the lower limbs indicates that differences in direction of overall translatory movement are not the primary source for the different interpretations (cf. Vanrie et al., 2004) and suggests that if the process of depth assignment depends on certain visual features, these features are not located in the lower part of the stimulus. Indeed, as in the previous experiment, it is the movement direction of the upper limbs, perpendicular, vis-à-vis the torso, that constitutes an important aspect for the facing bias to occur. However, as also demonstrated by the significant main effect of viewpoint and the significant interaction, a preferred interpretation was only found when the stimulus was presented from a $3 / 4$ viewpoint. This confirms the trend that can also be seen in the results of Experiment 2-namely, that a preference for a particular interpretation is either absent or much less pronounced for frontal viewpoints. This effect of viewpoint comprises a major difference between, on the one hand, the results of Experiments 2 and 3 and, on the other hand, the findings from Experiment 1 

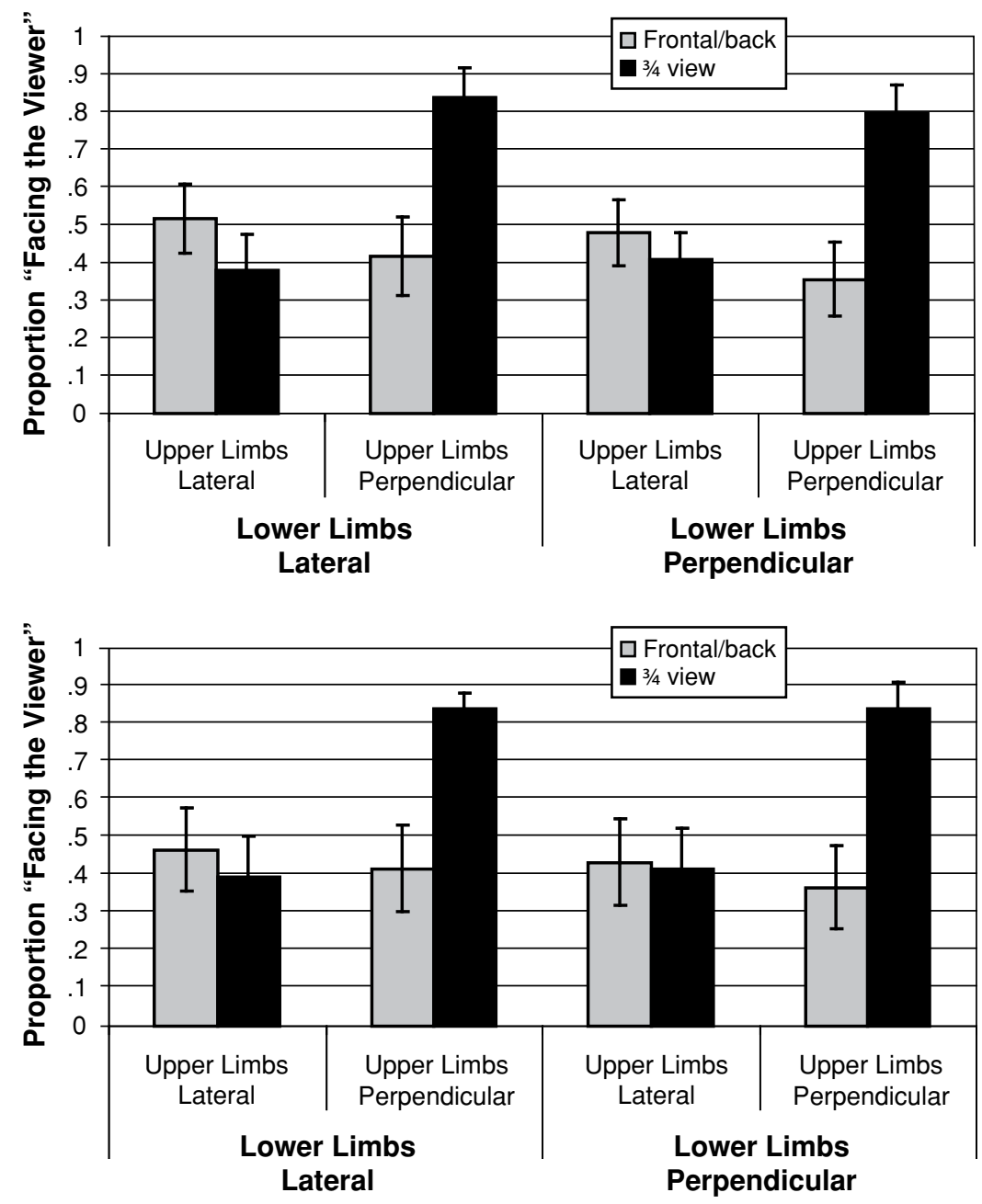

Figure 7. The proportions of responses in which observers in Experiment 3 reported the point-light figure in an orientation toward them for the frontal/back and $3 / 4$ views of the eight actions, classified in terms of the direction of movement (perpendicular or lateral) of the upper and lower limbs (error bars denote the standard error). The upper panel depicts the four stimuli that correspond to the first time segment of the stimuli used in Experiment 2, and the lower panel depicts the data for the four stimuli corresponding to the second time segment.

and those reported in Vanrie et al. (2004), in which no significant differential effect of viewpoint was observed. ${ }^{8}$ We hypothesize that the use of unfamiliar movements precluded any systematic effects of stored knowledge, but instead led observers to rely more on specific visual features. The assumption that such actions are primarily decoded in terms of their spatiotemporal characteristics, regardless of previously stored, semantic representations, is consistent with findings from neuroimaging studies (Decety et al., 1997; Grèzes, Costes, \& Decety, 1998) showing predominant activation of the dorsal pathway when people are presented with unfamiliar movements, in comparison with conditions in which meaningful actions are presented. Furthermore, a similar distinction has also been suggested from research on imitation and apraxia: Whereas meaningful actions can be imitated by both a semantically mediated route and a direct, visual route, the imitation of meaningless actions can only proceed through the latter (Goldenberg \& Hagmann, 1997; Rumiati \& Tessari, 2002; see also Hermsdörfer et al., 2001).

There is an alternative hypothesis concerning the effects of the different actions we observed in Experiments 2 and 3 , which we need to address. It could be the case that to our observers, the unfamiliar actions were not really that novel or completely devoid of semantic correlates or that (parts of) the unfamiliar actions might be similar to (parts of) actions for which a stored representation does exist. In both cases, the differences for the different actions, which we claim are due to the kinematic pattern influencing the process of depth assignment, could in fact be the result of a top-down factor. Indeed, we cannot exclude the possibility that for certain individual observers, some of 
the "unfamiliar" actions were mapped onto similar stored representations. However, we do not believe that this fully accounts for the data. First of all, we obtained independent evidence, which confirms that these actions are indeed less familiar and meaningful than the actions used in Experiment 2. We presented 10 new observers with the five views of nine actions (the four familiar actions used in Experiment 1, the four unfamiliar actions used in Experiment 2 , and the walking action) and asked them to rate, on an 11-point scale, how meaningful/meaningless and novel/familiar these actions appeared to them. The average rating for the familiar actions (rowing, jumping, saluting, cycling, and walking) was $9.06(S D=2.87)$ for the meaningful scale and 8.12 (3.01) for the familiarity scale. Both values were significantly higher than those for the unfamiliar actions: 4.69 (2.12) and 5.33 (2.87), respectively. Moreover, we also explicitly asked observers to provide a name or short description of each action, to provide another action that they thought resembled the visual appearance of the presented action (i.e., was similar in terms of the movements of the limbs), and to rate, on a 4-point scale, whether this resemblance was very small (1), small (2), strong (3), or very strong (4). In contrast to the familiar actions, the unfamiliar actions were only rarely labeled, but almost always described in terms of movements of upper and lower limbs. Moreover, the strength of the resemblance to other actions was generally rated as small $(M=1.87)$, and even for a single action, there was little consistency across observers concerning actions resembling the presented movement pattern. These data suggest that it is unlikely that systematic mappings onto stored action representations would have resulted in the regular pattern of results we obtained in Experiments 2 and 3. Second, the differential effect of viewpoint also argues against this alternative hypothesis. If parts of the unfamiliar action were indeed "recognized" and, because of their similarity to (parts of) a stored action representation, preferentially interpreted in a particular way, there is no straightforward reason why this would depend on the presented viewpoint. In light of these elements, we believe that the different results obtained for the different actions in Experiments 2 and 3 can be better accounted for by differences in kinematic features.

\section{GENERAL DISCUSSION}

In three experiments, observers indicated the global depth orientation of ambiguous point-light figures. By manipulating the spatiotemporal characteristics of the point lights, we showed that (1) point-light figures without ordinal depth cues are generally bistable, regardless of their kinematic pattern, and (2) the perception of the global orientation is strongly affected by aspects of the movement pattern.

In Figure 8, we sketch the situation in which the visual system processes an ambiguous point-light figure. From previous research with a point-light walker, it is known that this ambiguous stimulation leads to one of two possible percepts and that there is a difference in the frequen-

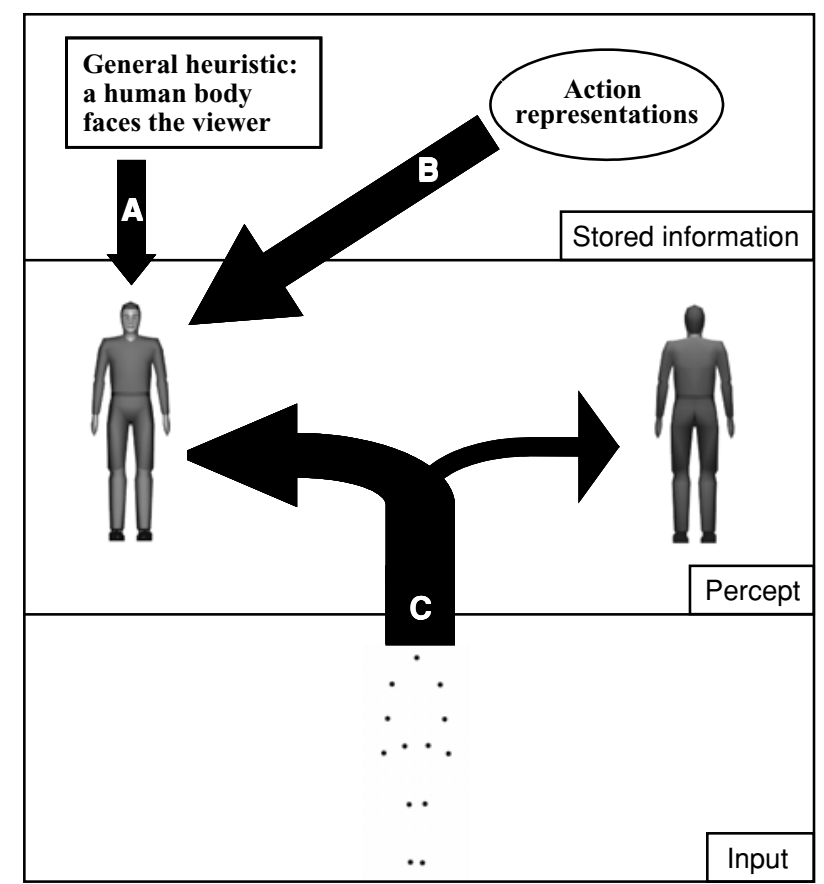

Figure 8. Sketch of the components thought to be involved in the process of depth assignment in ambiguous point-light figures. (A) A general preference to interpret a human body as being oriented toward the viewer can lead to a facing bias. (B) Stored action representations can affect the final interpretation. (C) Processing of the spatiotemporal characteristics of the point-light stimulus itself can favor a particular percept.

cies with which the two percepts are reported. First of all, the present study indicates that these two possible percepts are also retained for other point-light actions. All movement patterns that have been studied here and previously in Vanrie et al. (2004) give rise to (at least) two different interpretations, establishing the point-light figure as a genuine bistable configuration. Moreover, the difference in frequency for the two percepts was again observed, indicating that this phenomenon is not limited to a walking motion. However, it was also found that the ratios of "facing the viewer"/"facing away" responses strongly differ for different actions, suggesting that the resolution of the depth order ambiguity is not simply an isolated, stochastic process. In the following paragraphs, we discuss three components [(1) a general heuristic, (2) stored action representations, and (3) kinematic aspects of the stimulus itself; see Figure 8] that we assume to be relevant to this process of depth assignment, and indicate how the present results relate to them.

1. General heuristic. Similarly to, for example, the tendency to perceive a Necker cube as if it is located below eye level (see, e.g., Bisiach, Ricci, Lai, De Tanti, \& Inzaghi, 1999) or the general assumption that a light source is located above an object (see, e.g., Enns \& Rensink, 1990), observers may employ a general heuristic to perceive human bodies as facing the viewer. Consistent with this hypothesis is the finding of Vanrie et al. (2004) 
that not only standard point-light walkers but also pointlight figures walking backward are preferentially interpreted as being oriented toward the viewer. The present study does not necessarily contradict the existence of such a tendency, but the fact that the facing bias was not found for all actions does indicate that it is not the only determining factor when interpreting ambiguous point-light actions.

2. Stored action representations. It is almost certainly the case that some top-down components are implicated in the perception of point-light figures. Several studies have already provided convincing evidence for a role for stored knowledge and top-down effects in the perception of human (point-light) actions and body postures (see, e.g., Bülthoff et al., 1998; Cavanagh et al., 2001; McBeath et al., 1992; Shiffrar \& Freyd, 1990, 1993; Thornton, Rensink, \& Shiffrar, 2002). For example, Bülthoff et al. showed that stereoscopically presented, depth-scrambled point-light figures were nevertheless interpreted as normal walkers, suggesting strong top-down feedback. Somewhat similarly, Vanrie et al. (2004) showed that the bias to perceive a point-light walker as facing the viewer was sufficient to occasionally override an occlusion cue that indicated the veridical depth orientation. The present study was not explicitly focused on the influence of stored action representations, but the discrepancies between the results for the familiar (Experiment 1) and unfamiliar (Experiments 2 and 3) actions do also point in that direction. Assuming that top-down feedback, in this case, from stored action representations, is able to guide processing and stabilize ambiguous percepts (Cavanagh et al., 2001; Grossmann \& Dobbins, 2003), one would expect this to occur in a similar manner across different views of the same action. On the other hand, when such a stabilizing factor is absent and observers have to rely primarily on the information available at the input level, then differences in this information-for example, due to changes in the presented viewpoint - become much more important. This is also the pattern of results that was found in the present study: For the familiar actions, no systematic effects of viewpoint were observed, whereas such an effect was observed for the unfamiliar actions.

3. Kinematic aspects. Previous research has demonstrated that the perception of depth in point-light walkers is sensitive to depth cues available in the visual information presented to the system (Vanrie et al., 2004). The present results further emphasize the importance of this information for the process of depth assignment, by showing that not only explicit depth cues but also the perceived body articulation affects the perceived depth orientation. The intriguing question remains, of course, how, exactly, these visual features - which in themselves are also ambiguous and never indicate a unique percept - can determine perceived depth order. The data indicate that the direction of movement of the upper limbs constitutes the differentiating feature here but, unfortunately, they do not provide a definitive answer regarding the underlying mechanism.

Nevertheless, we want to offer some possibilities, although they are speculative, as to how such a phenomenon could come about. First, the arms may function as a figure on the background of the torso. The four dots making up the torso (i.e., the two shoulder and two hip points) form a closed contour, which constitutes a relatively fixed configuration moving about as a single unit. When the arms move within the area delineated by these four dots - which only happens with perpendicular arm movements - some form of local, motion-induced figure-ground segmentation may take place, in which case the arms would become the figure, and hence, be seen as closer. This does not occur, however, in a frontal/back view nor in any of the views of the actions with lateral arm movements, because the wrist/elbow point lights never enter this specific area. That is, with these views, no salient figure-ground segmentation takes place, and hence, no bias is observed. In addition, the lack of such an implicit background surface might also explain why movements of the lower limbs are not affecting the interpretation. Testing this account could be done by lowering the probability of a figure-ground segregation - for example, by removing the hip or shoulder dots, thereby destroying the closed contour implying a surface. A second possibility has already been mentioned in the introduction of Experiment 2: It could be the case that the 3-D motion of a single limb (Webb \& Aggarwal, 1982 ) is tracked, on the basis of which the 3-D orientation of the entire body is reconstructed (cf. Kawabata, 1986). Because the wrist/elbow dots are located near the center of the figure and exhibit the largest displacements in these actions, they are more likely to become the prime targets for initial tracking (and not the lower limbs). If the 3-D trajectory of the initially tracked limb (i.e., one of the arms) were to then be preferentially perceived as coming toward the viewer instead of moving away, the subsequent reconstruction of the entire body would automatically result in a higher probability of the corresponding interpretation. In other words, the higher sensitivity and bias for approaching objects that has already been observed in the visual system (Lewis \& McBeath, 2004; Shirai \& Yamaguchi, 2004) does not operate on the level of the point-light figure (as evidenced by the results of Experiment 3 and of Vanrie et al., 2004), but it might still operate on a more local scale - for example, on the level of individual dots. Because only the actions with perpendicular arm movements have this moving toward/moving away ambiguity of the upper limbs, the facing bias is found only for these actions. Of course, the plausibility of such a hypothesis would depend heavily on its ability to account for why this bias does not occur when the figure is seen from a frontal viewpoint. Nevertheless, the important issue here is that stored action representations or semantic influences do not need to be invoked to explain the results for either of these two hypotheses. Indeed, the data we obtained by using the unfamiliar actions indicate that these topdown components are not the (only) determining factors, and that purely kinematic aspects, such as the plane of movement of certain limbs, can influence perceived depth orientation.

Finally, now that we have discussed the three components (general heuristic, stored action representations, and 
kinematic aspects) separately, we should stress that they are, of course, not mutually exclusive, and most likely also interact. One example of such an interaction, and possibly one of the mechanisms underlying the results observed here, would be when the visual system uses occlusion violations to resolve the ambiguity. Because no occlusion was applied in the stimuli used here, the dots remained visible even when they would have been occluded if we had been using a solid body; the point-light stimulus permits a violation of the rules of occlusion that are normally imposed by the physical world. For some actions, the two possible interpretations (facing the viewer and facing away) differ in the number of occlusion violations they generate, leading the visual system to preferentially select the interpretation with the smallest number of violations. In this scenario, the interaction between stored knowledge of the structure of the human body, which is known to affect visual perception of actions (see, e.g., Shiffrar \& Freyd, 1990, 1993), and the specific articulations by the point-light figure will lead to different distributions of reported percepts for different movement patterns. Compared with the previous two hypotheses, in which a facing bias could be explained by making an assumption about the processing of the (3-D) trajectories of the dots, here, the perceived structure and articulation of the body are crucial. To illustrate: Whereas the removal of, for example, the shoulder dots would have consequences according to the figure-ground hypothesis, as sketched above, the occlusion violation hypothesis would not predict such an effect, because although this removal would indeed affect processes on a lower level, no changes take place in terms of human body structure. One way to explicitly investigate the occlusion violation hypothesis would be to employ actions in which using these occlusion violations would lead to a "facing away" interpretation (e.g., some action pattern in which the arms move not in front, but behind the torso).

The perception of objects as 3-D entities has received a great deal of attention, even for the specific case of classic structure-from-motion stimuli (see, e.g., Andersen \& Bradley, 1998; Hol, Koene, \& van Ee, 2003). Somewhat surprisingly, perhaps, the corresponding aspect of depth perception in point-light actions has received little or no attention. Nevertheless, as a whole, the data presented here indicate that the interpretation of a point-light figure in terms of its global depth orientation results from a fairly complex and flexible process involving both bottom-up and top-down components. Although more research is needed to determine the precise nature and contributions of these components and how, exactly, this process of depth assignment and the perception of the global orientation relates to the perception and identification of (point-light) actions in general, and how intertwined these processes are, the issue of depth perception seems to constitute an essential component in the observation of point-light actions.

In sum, everyday life provides ample cues as to the depth orientation of human bodies. When these cues are unavailable or not unequivocal, however, the visual system is sensitive to the ensuing ambiguity and, instead of employing a fixed heuristic to interpret all human bodies in a particular manner, takes into account the kinematics of the body to resolve it. We propose that perceiving depth in point-light figures results from a flexible process in which both bottom-up and top-down components play a role. We argue that theoretical or computational modelers striving to provide an exhaustive account of biological-motion or action perception will need to explicitly incorporate this often ignored issue of depth perception in point-light actions.

\section{REFERENCES}

Ahlström, V., Blake, R., \& Ahlström, U. (1997). Perception of biological motion. Perception, 26, 1539-1548.

Andersen, R. A., \& Bradley, D. C. (1998). Perception of threedimensional structure from motion. Trends in Cognitive Sciences, 2 , 223-228.

Attneave, F. (1971). Multistability in perception. Scientific American, 225, 62-71.

Bernstein, L. J., \& CoOPER, L. A. (1997). Direction of motion influences perceptual identification of ambiguous figures. Journal of Experimental Psychology: Human Perception \& Performance, 23, 721-737.

Bisiach, E., Ricci, R., Lai, E., De Tanti, A., \& InZaghi, M. G. (1999). Unilateral neglect and disambiguation of the Necker cube. Brain, 122, 131-140.

Bülthoff, I., Bülthoff, H., \& Sinha, P. (1998). Top-down influences on stereoscopic depth-perception. Nature Neuroscience, 1, 254-257.

Cavanagh, P., Labianca, A. T., \& Thornton, I. M. (2001). Attentionbased visual routines: Sprites. Cognition, 80, 47-60.

Cutting, J. E., \& Kozlowski, L. T. (1977). Recognizing friends by their walk: Gait perception without familiarity cues. Bulletin of the Psychonomic Society, 9, 353-356.

Decety, J., Grèzes, J., Costes, N., Perani, D., Jeannerod, M., PROCYK, E., ET AL. (1997). Brain activity during observation of actions: Influence of action content and subject's strategy. Brain, 120, 1763-1777.

Dekeyser, M., Verfaillie, K., \& Vanrie, J. (2002). Creating stimuli for the study of biological-motion perception. Behavior Research Methods, Instruments, \& Computers, 34, 375-382.

DitTRich, W. H. (1993). Action categories and the perception of biological motion. Perception, 22, 15-22.

Domini, F., \& CAUDEK, C. (2003). 3-D structure perceived from dynamic information: A new theory. Trends in Cognitive Sciences, 7, 444-449.

EnNS, J. T., \& RENSINK, R. A. (1990). Influence of scene-based properties on visual search. Science, 247, 721-723.

Giese, M. A., \& PogGio, T. (2003). Neural mechanisms for the recognition of biological movements. Nature Reviews Neuroscience, 4, 179-192.

GoldenberG, G., \& Hagmann, S. (1997). The meaning of meaningless gestures: A study of visuo-imitative apraxia. Neuropsychologia, 35, 333-341.

Grèzes, J., Costes, N., \& Decety, J. (1998). Top-down effect of strategy on the perception of human biological motion: A PET investigation. Cognitive Neuropsychology, 15, 553-582.

Grossmann, J. K., \& Dobbins, A. C. (2003). Differential ambiguity reduces grouping of metastable objects. Vision Research, 43, 359-369.

Hermsdörfer, J., Goldenberg, G., Wachsmuth, C., Conrad, B., Ceballos-Baumann, A. O., Bartenstein, P., et al. (2001). Cortical correlates of gesture processing: Clues to the cerebral mechanisms underlying apraxia during the imitation of meaningless gestures. NeuroImage, 14, 149-161.

Hogervorst, M. A., \& Eagle, R. A. (1998). Biases in three-dimensional structure-from-motion arise from noise in the early visual system. Proceedings of the Royal Society of London: Series B, 265, 15871593.

Hol, K., Koene, A., \& van Ee, R. (2003). Attention-biased multi-stable surface perception in three-dimensional structure-from-motion. Journal of Vision, 3, 486-498. 
Johansson, G. (1973). Visual perception of biological motion and a model for its analysis. Perception \& Psychophysics, 14, 201-211.

Kawabata, N. (1986). Attention and depth perception. Perception, 15, 563-572.

Lewis, C. F., \& McBeath, M. K. (2004). Bias to experience approaching motion in a three-dimensional virtual environment. Perception, 33, 259-276.

Mamassian, P., \& Landy, M. S. (1998). Observer biases in the 3D interpretation of line drawings. Vision Research, 38, 2817-2832.

McBeath, M. K., Morikawa, K., \& Kaiser, M. K. (1992). Perceptual bias for forward-facing motion. Psychological Science, 3, 362-367.

Moeslund, T. B., \& Granum, E. (2000, June). 3D human pose estimation using $2 D$-data and an alternative phase space representation. Paper presented at the IEEE Workshop on Human Modeling, Analysis and Synthesis, Hilton Head Island, SC.

Proffitt, D. R., Bertenthal, B. I., \& Roberts, R. J., JR. (1984). The role of occlusion in reducing multistability in moving point-light displays. Perception \& Psychophysics, 36, 315-323.

Rumiati, R. I., \& Tessari, A. (2002). Imitation of novel and wellknown actions: The role of short-term memory. Experimental Brain Research, 142, 425-433.

ShIFFRAR, M., \& FreYd, J. J. (1990). Apparent motion of the human body. Psychological Science, 1, 257-264.

Shiffrar, M., \& FreYd, J. J. (1993). Timing and apparent motion path choice with human body photographs. Psychological Science, 4, 379384.

Shirai, N., \& Yamaguchi, M. K. (2004). Asymmetry in the perception of motion-in-depth. Vision Research, 44, 1003-1011.

Thornton, I. M., Rensink, R. A., \& Shiffrar, M. (2002). Active versus passive processing of biological motion. Perception, 31, 837-853.

Vanrie, J., Dekeyser, M., \& Verfaillie, K. (2004). Bistability and biasing effects in the perception of ambiguous point-light walkers. Perception, 33, 547-560.

VAnRIE, J., \& Verfaillie, K. (2004). Perception of biological motion: A stimulus set of human point-light actions. Behavior Research Methods, Instruments, \& Computers, 36, 625-629.

Verfaillie, K., De Troy, A., \& Van Rensbergen, J. (1994). Transsaccadic integration of biological motion. Journal of Experimental Psychology: Learning, Memory, \& Cognition, 20, 649-670.

WebB, J. A., \& AgGarwal, J. K. (1982). Structure from motion of rigid and jointed objects. Artificial Intelligence, 19, 107-130.

\section{NOTES}

1. The extreme version of such an account would be that the visual system simply uses a default value: Confronted with a human body, the visual system assumes it is oriented toward the viewer (unless, of course, there is evidence to the contrary; see Vanrie et al., 2004). In fact, the assumption that the body is facing the viewer, or the camera, is one that has also been made in models of computer vision dealing with the recognition of bodies in motion (see, e.g., Moeslund \& Granum, 2000). Of course, such an extreme account cannot readily explain the fact that the ambiguous point-light figure does give rise to two interpretations.

2. These actions are visually "loopable," meaning that there are no abrupt visual changes in the transition from the last frame of the animation back to the first.
3. The pattern of results was very similar for the correct and the incorrect trials. The overall proportions of "facing the viewer" responses across nonlateral views for the four actions - separately for trials in which a correct orientation was reported and trials in which an incorrect orientation was reported - were .63 (correct trials) versus .64 (incorrect trials), .83 versus $.81, .91$ versus 1.00 , and .39 versus .38 for jumping, rowing, cycling, and saluting, respectively. On a related note, because observers do sometimes perceive nonveridical orientations, the term "multistable" would actually be a more precise label for these point-light actions. However, because the primary focus here is the perception of one of the two possible depth orders and not so much the specific view ( $3 / 4$ or frontal/back), we prefer the term bistable in this context.

4. Unless indicated otherwise, we will use the term kinematics to refer to both the specific movements of a human body as well as to the trajectories of the dots comprising the point-light figure. These are two distinct concepts, but in the present context, their scopes overlap almost completely.

5. Again, most errors were due to an inability to distinguish between the $3 / 4$ and frontal/back views. However, it is also true that the patterns of results with regard to "facing the viewer" versus "facing away" responses were quite similar for correct and incorrect trials. The proportions of "facing the viewer" responses for the $3 / 4$ views of the four actions for correct and incorrect trials were, respectively, .34 (correct trials) versus .53 (incorrect trials), .94 versus $.93, .42$ versus .61 , and .95 versus .92 .

6. For example, in Experiment 2, it was found that differences in the plane of movement of the lower limbs do not significantly alter the interpretation. One possibility for why no significant differences were found between the lateral and orthogonal lower limb movements is that the results for the orthogonal movements were confounded due to the fact that the first step was taken forward and the second step backward. Although we do not believe this affected the results (because previous research has already indicated equivalent results for point-light figures walking backward and forward; Vanrie et al., 2004), the present setup can provide a more strict empirical test.

7. Distinguishing between the $3 / 4$ and frontal/back views was even more difficult in the present experiment, but again, the patterns of results with regard to "facing the viewer" versus "facing away" responses were quite similar for correct and incorrect trials. The proportions of "facing the viewer" responses for the $3 / 4$ view of the four major action types for the correct and incorrect trials were .25 (correct trials) versus .40 (incorrect trials), .90 versus $.82, .34$ versus .44 , and .86 versus .78 .

8 . Although the interaction between the action and the presented viewpoint in Experiment 1 was marginally significant, there still seems to be a qualitative difference between the systematic effect in Experiments 2 and 3 and the more variable effect of viewpoint in Experiment 1.

(Manuscript received January 14, 2005; revision accepted for publication July 18, 2005.) 